\title{
Visualization of Minkowski patch
}

\section{Rania Bahgat Mohamed Amer}

Department of Physics and Mathematics Engineering, Faculty of Engineering/ P.O. 44519/ Zagazig University, Egypt

\section{Email address:}

dr.raniaamer@authorname.com

\section{To cite this article:}

Rania Bahgat Mohamed Amer. Visualization of Minkowski Patch. Pure and Applied Mathematics Journal. Vol. 3, No. 6, 2014, pp. $132-136$. doi: 10.11648/j.pamj.20140306.14

\begin{abstract}
This study is an introduction to visualize Minkowskian ( $n, 1)$ geometry for all $\mathrm{n} \geq 1$. The Minkowski geometry naturally encodes the ideas of inertial frames, time and space dilation. Moreover, it also includes studying Minkowski patch which is the natural structure of Minkowski space.
\end{abstract}

Keywords: Minkowski Space, Einstein Space, Minkowski Patch, Improper Point, Crooked Surface

\section{Introduction}

The Universe contains many kinds of spaces and types of different curvatures. The space scientists have hypothesized many theories to explain the shape and nature of the Universe. Minkowski space is one the newly hypothesized geometrical spaces that explains many unknown phenomena in the Universe. Minkowski Space, which named by Hermann Minkowski, is mathematical setting in which Einstein's theory of special relativity is most conveniently formulated by 1907 Minkowski realized that the special theory of relativity based on Lorentz and Poincare, could be best understood in a ndimensional space. Studying Minkowskian mathematical models will enhance our understanding of space-time and the Universe. Moreover, the construction of fundamental properties of Minkowski space develops and enhances techniques in space with applications in general and special relativity. Also, this study will help Egyptian mathematical scientists to understand and integrate effectively in space studies.

\section{Minkowski Space}

Definition 1. Minkowski Space is simply connected, geodesically complete, flat Lorentzian manifold.

Definition 2. An affine space is defined as a pair (A, V) consisting of a set $A$ together with a vector space $V$, such that $\mathrm{V}$ acts freely and transitively group action on $\mathrm{A}$. There is a map $\tau$ called translation by the vector $\mathrm{v}$,

$$
\tau: V x A \rightarrow A, A:(v, a) \rightarrow v+a
$$

with the following properties:

Identity

$$
\forall \mathrm{a} \in \mathrm{A}, 0+\mathrm{a}=\mathrm{a} \text { or } \mathrm{a}+0=\mathrm{a} .
$$

Associatively

$$
\forall \mathrm{v}, \mathrm{w} \in \mathrm{V}, \forall \mathrm{a} \in \mathrm{A}, \mathrm{v}+(\mathrm{w}+\mathrm{a})=(\mathrm{v}+\mathrm{w})+\mathrm{a}
$$

Uniqueness

$$
\forall \mathrm{a} \in \mathrm{A}, \mathrm{V} \rightarrow \mathrm{A}: \mathrm{v} \rightarrow \mathrm{v}+\mathrm{a} .
$$

The action is free

$$
\forall \mathrm{V} \in \mathrm{V} \exists \text { a point } \mathrm{a} \in \mathrm{A}: \mathrm{a}+\mathrm{v}=\mathrm{a} \text { then } \mathrm{v}=0 .
$$

The action is transitive

$$
\forall \mathrm{a}, \mathrm{b} \in \mathrm{A} \exists \text { a vector } \mathrm{v} \in \mathrm{V}: \mathrm{b}=\mathrm{a}+\mathrm{v} .
$$

The dimension of $\mathrm{A}$ is the dimension of the vector space of translations V. Then the vector space of translations is the space of vectors in the plane or in space [1].

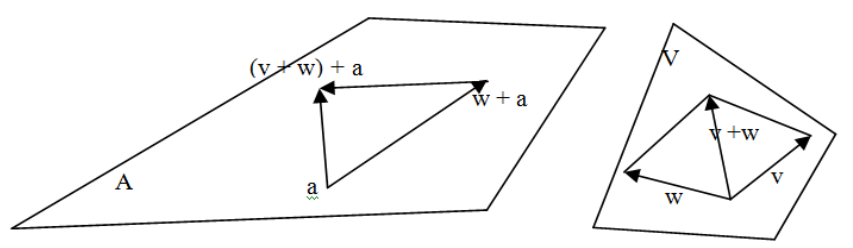

Figure 1 Points and displacements in an affine space 


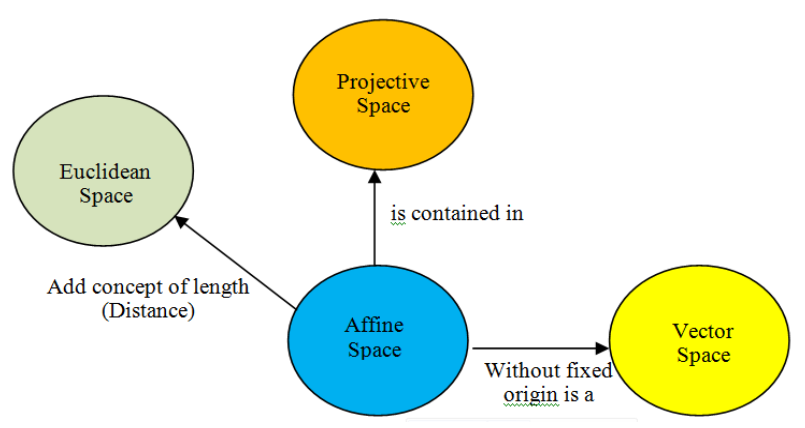

Figure 2. The relation between affine space and other spaces

Theorem 1. Minkowski space is an affine space whose underlying vector space of signature $(\mathrm{n}, 1)$.

Proof:

Let $\mathrm{E}$ denote an affine space which is different from real vector space $\mathrm{V}=R^{n, 1}$ by having no special or fixed origin. Let the translation group $\mathrm{V}=R^{n, 1}$ is given the structure of non degenerate symmetric bilinear form of index 1 . The inner product space $\mathrm{R}^{\mathrm{n}+1,2}$ is introduced by symmetric bilinear form of type $(n+1,2)$ as $I_{n+1} \oplus-I_{2}$

$$
\langle\mathrm{v}, \mathrm{v}\rangle=\mathrm{v}_{1}^{2}+\mathrm{v}_{2}^{2}+\ldots \ldots \ldots \ldots+\mathrm{v}_{\mathrm{n}+1}^{2}-\mathrm{v}_{\mathrm{n}+2}^{2}-\mathrm{v}_{\mathrm{n}+3}^{2},
$$

for all $v \in V$. [7]:

The bilinear form on inner product space $R^{n, 1}$ is written as

$$
B(u, v)=u_{1} v_{1}+u_{2} v_{2}+u_{3} v_{3}+\ldots+u_{n} v_{n}-u_{n+1} v_{n+1}
$$

for all $\mathrm{u}, \mathrm{v} \epsilon \mathrm{V}$

The bilinear form is defined a lorentz metric on $\mathrm{E}$ as

$$
\mathrm{ds}^{2}=\mathrm{dx}_{1}^{2}+\mathrm{dx}_{2}^{2}+\mathrm{dx}_{3}^{2}+\cdots \ldots+\mathrm{dx}_{\mathrm{n}}^{2}-\mathrm{dx}_{\mathrm{n}+1}^{2}
$$

which is invariant under the translation of $\mathrm{E}$.

So Minkowski $(n+1)$-space is denoted by $E^{n, 1}$ is a real affine space of dimension $\mathrm{n}+1$ with a non degenerate inner product of index ' 1 '.

The geodesics in Minkowski space $E^{n, l}$ are Euclidean straight line of the form:

$$
\mathrm{R} \stackrel{\gamma}{\rightarrow} \mathrm{E}^{\mathrm{n}, 1}, \mathrm{t} \rightarrow \mathrm{p}_{0}+\mathrm{tu},
$$

Where a point on a line $\mathrm{p}_{0} \in \mathrm{E}^{\mathrm{n}, 1}$, a vector $\mathrm{u} \in \mathrm{R}^{\mathrm{n}, 1}$ is space-like, time-like, or null and a real parameter $t \in R$. [3]:

Then there are three types of curves in Minkowski space

1- Light-like curves form a cone in Minkowski space and dividing it into two parts so the light like curves that pass through the event will be on the surface of the light cones.

2-Time-like curves fall within a cone defined by light-like curves so the time like curves that pass through the event will remain inside that event's past and future light cones.

3-Space-like curves that pass through the event will be outside the light cones.
Definition 3. A vector $\mathrm{u}=\left[\begin{array}{c}u_{1} \\ u_{2} \\ u_{3} \\ \cdots \cdots \\ u_{n}\end{array}\right] \in \mathrm{R}^{\mathrm{n}, 1}$ is said to be:

- spacelike if

$$
B(u, u)=u_{1}^{2}+u_{2}^{2}+\cdots \ldots+u_{n}^{2}-u_{n+1}^{2}>0,
$$

- lightlike (or null) if

$$
\mathrm{B}(\mathrm{u}, \mathrm{u})=\mathrm{u}_{1}^{2}+\mathrm{u}_{2}^{2}+\cdots \ldots+\mathrm{u}_{\mathrm{n}}^{2}-\mathrm{u}_{\mathrm{n}+1}^{2}=0,
$$

- timelike if

$$
\mathrm{B}(\mathrm{u}, \mathrm{u})=\mathrm{u}_{1}^{2}+\mathrm{u}_{2}^{2}+\cdots \ldots+\mathrm{u}_{\mathrm{n}}^{2}-\mathrm{u}_{\mathrm{n}+1}^{2}<0 .
$$

The union of all null lines is called the lightcone. Then the light-cone with vertex $\mathrm{p}$ of Minkowski space $\mathrm{E}^{\mathrm{n}, 1}$ is defined as the set of all lightlike vectors of $\mathrm{E}^{\mathrm{n}, 1}$, that is,

$\mathrm{L}(\mathrm{p})=\left\{\left(\mathrm{x}_{1}, \mathrm{x}_{2}, \ldots, \mathrm{x}_{\mathrm{n}}\right) \in \mathrm{E}^{\mathrm{n}, 1} ;\left\{\mathrm{x}_{1}^{2}+\mathrm{x}_{2}^{2}+\cdots \ldots+\mathrm{x}_{\mathrm{n}}^{2}-\mathrm{x}_{\mathrm{n}+1}^{2}\right.\right.$
$=0\}-\{(0,0,0)\}[4]$.

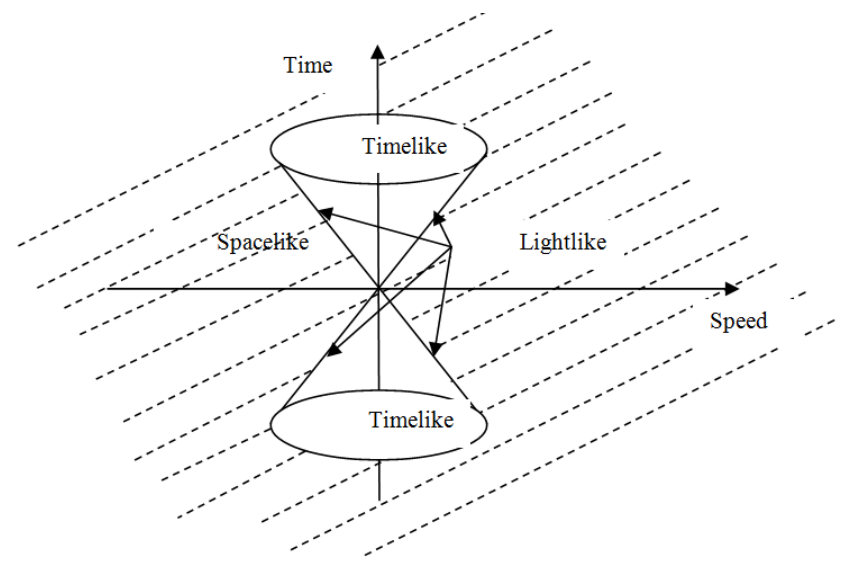

Figure 3. The curves in Minkowski space

\section{Minkowski Patch}

Einstein space [5] of dimension $\mathrm{n}$ is denoted by $\operatorname{Ein}^{\mathrm{n}}$ is a homogenous space and it is the image of null cone

$$
\mu^{\mathrm{n}, 1}=\left\{\mathrm{u} \in \mathrm{R}^{\mathrm{n}, 1} \mid<u, u>=0\right\}-\{0\} ;
$$

under projection

$$
\mathrm{R}^{\mathrm{n}, 1}-\{0\} \rightarrow \mathrm{RP}^{\mathrm{n}+1}
$$

This mean that Einstein space is a conformal compactness of Minkowski space.

The double covering $\widehat{E l n^{n}}$ is a quotient of null cone $\mu^{\mathrm{n}, 1}$ by the action of positive scalar multiplications.

The Minkowski patch Min ( $p$ ) determined by an element $p$ of $\operatorname{Ein}^{n}$ is the complement of the light cone L(p) in $\operatorname{Ein}^{n}$, i.e, $\operatorname{Ein}^{n}-L(p)$ and has the natural structure of Minkowski space $\mathrm{E}^{\mathrm{n}, 1}[5]$. 


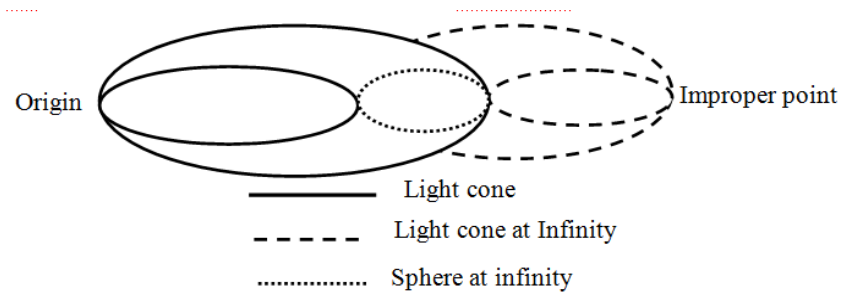

Figure 4. The points in Minkowski space

From figure 4 we see that the origin or the vertex with homogeneous coordinates $\left[\begin{array}{c}0_{\mathrm{n}} \\ 0 \\ 1\end{array}\right]$ is mapped to the improper point having homogeneous coordinates $\left[\begin{array}{c}0_{\mathrm{n}} \\ 1 \\ 0\end{array}\right]$, where a zero vecor $0_{n} \in R^{n}$.

Let $\mathrm{p}_{\infty}$ be an improper point, in the double-covering $\widehat{\operatorname{Eln}^{n}}$, every Minkowski patch are attached two improper points:

1- The spatial improper point has homogeneous coordinates $\left[\begin{array}{c}0 \\ -1 \\ 0_{n}\end{array}\right]$ and it is denoted by the unique element $p_{\infty}^{s p}$ such that the given Minkowski patch is $\operatorname{Min}\left(\mathrm{p}_{\infty}^{\mathrm{sp}}\right)$.

2 - The timelike improper point has homogeneous coordi nates $\left[\begin{array}{l}0 \\ 1 \\ 0_{\mathrm{n}}\end{array}\right]$ and it is denoted by the unique element $\mathrm{p}_{\infty}^{\mathrm{ti}}$ such that the given Minkowski patch is $\operatorname{Min}+\left(\mathrm{p}_{\infty}^{\mathrm{ti}}\right)$.

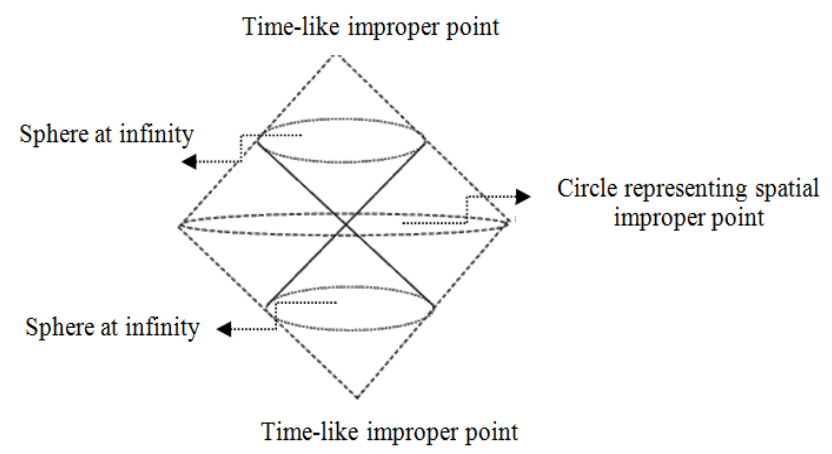

Figure 5. The improper points in Minkowski patch of double covering $\widehat{\text { Enn }^{n}}$

The point of view of one Minkowski patch is shown in Figure 5 where the timelike improper point is represented by two identified points, the spatial improper point is represented by an entire circle and the sphere at infinity is represented by the two identified circles. A point on one sphere is identified to a point on the other sphere by scalar multiplication by -1 .

Theorem 2. The crooked surfaces have four points and Minkowski space $E^{\mathrm{n}, 1} \in \operatorname{Min}\left(\mathrm{p}_{1}\right) \cup \operatorname{Min}\left(\mathrm{p}_{2}\right) \cup \operatorname{Min}\left(\mathrm{p}_{0}\right) \cup$ $\operatorname{Min}\left(\mathrm{p}_{\infty}\right)$.

Proof:

In crooked surfaces $\in \mathrm{R}^{\mathrm{n}}$, we get four points in a stem configuration which is defined as the union of null lines passes through the vertex (the origin) inside the light cone:

1 - The vertex point $\mathrm{p}_{0}=\left[\begin{array}{c}0_{\mathrm{n}} \\ 0 \\ 1\end{array}\right]$,
2- Two ideal points: $\mathrm{p}_{1}=\left[\begin{array}{l}0 \\ 1 \\ 0_{\mathrm{n}}\end{array}\right], \quad \mathrm{p}_{2}=\left[\begin{array}{c}0 \\ -1 \\ 0_{\mathrm{n}}\end{array}\right]$,

3- The improper point $\mathrm{p}_{\infty}=\left[\begin{array}{c}0_{\mathrm{n}} \\ 1 \\ 0\end{array}\right]$.

We get 8 segments between $\left(\mathrm{p}_{0}, \mathrm{p}_{1}\right),\left(\mathrm{p}_{0}, \mathrm{p}_{2}\right),\left(\mathrm{p}_{\infty}, \mathrm{p}_{1}\right)$ and $\left(\mathrm{p}_{\infty}, \mathrm{p}_{2}\right)$ and the complement of $\operatorname{Ein}^{\mathrm{n}}-\left[\mathrm{L}\left(\mathrm{p}_{0}\right) \cup \mathrm{L}\left(\mathrm{p}_{\infty}\right)\right]$ consists of four quadrilateral regions. Then $\mathrm{E}^{\mathrm{n}, 1} \in \operatorname{Min}\left(\mathrm{p}_{1}\right) \cup$ $\operatorname{Min}\left(\mathrm{p}_{2}\right) \cup \operatorname{Min}\left(\mathrm{p}_{0}\right) \cup \operatorname{Min}\left(\mathrm{p}_{\infty}\right)$ and the proof is complete.

\subsection{Visualized Minkowski Patch}

Let $\tilde{\mathrm{p}} \in \operatorname{Ein}^{\mathrm{n}} \approx \mathrm{R}^{\mathrm{n}+1} \backslash\{0\}$ and the image of null geodesics $\alpha$ in $\operatorname{Ein}^{\mathrm{n}}$ are spiral curves in $\mathrm{R}^{\mathrm{n}+1} \backslash\{0\}$. So that the light cone is the union of geodesics contain $\tilde{p}$. Then, if $n=2$, the light cone of an element $\tilde{p}$ of $\operatorname{Ein}^{2}$ is a singular surface of revolution in $\mathrm{R}^{3}$ obtained by rotating a spiral contained in a vertical 2-plane around an axis of the plane. From fig. 6 we can visualize how Minkowski patches embed in $\mathrm{R}^{3} \backslash\{0\}$.

From fig. 6 we can deduced that Minkowski patch $\operatorname{Min}^{+}(\tilde{p})$ in $\operatorname{Ein}^{\mathrm{n}}$ is the intersection between the future oriented time like curve $\mathrm{I}+(\tilde{\mathrm{p}})$ and the past oriented time like curve I $-\left(\alpha^{2}(\tilde{\mathrm{p}})\right)$.

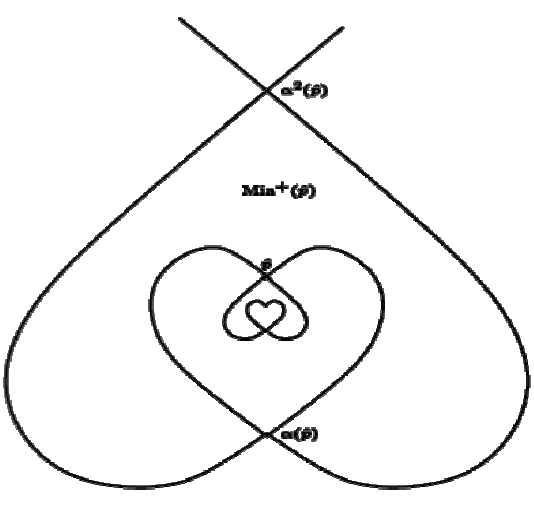

Figure 6. Minkowski patch in $\operatorname{Ein}^{2} \approx R^{3}-\{0\}$

\section{The Closure}

Definition 4. Crooked plane is a polyhedral surface in the Einstein space consists of wings which are two null planes inside the light cone, the stem is the union of two quadrants of two null lines (time-like lines) inside the light cone and the spine is a space-like vector [6].

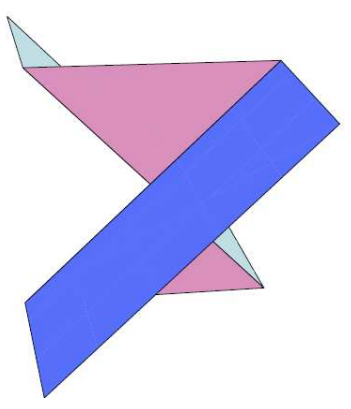

Figure 7. A crooked plane

From fig. 7 we can see the wings are half planes tangent to 
the light cone and the stem are the two infinite timelike triangles.

Definition 5. A set has the closure property under a particular operation if the result of the operation is always an element in the set

1. The light cone $\mathrm{L}(\mathrm{p})$ is the closure of null plane containing $\mathrm{p}$.

2. The closure of a stem $\overline{S(C)}$ is two light-like geodesics.

3. The closure of each wings $\mathrm{W}_{1}, \mathrm{~W}_{2}$ intersect ideal light cone $\mathrm{L}\left(\mathrm{p}_{\infty}\right)$.

4. The closure of time-like geodesic and space-like geodesic are time-like circle and space-like circle containing po.

5. Crooke plane $\mathrm{C}$ is homomorphic to $\mathrm{R}^{2}$ and the complement $\mathrm{E}^{2}, 1-\mathrm{C}$ consists of components each homomorphic to $\mathrm{R}^{3}$. This mean crooked half spaces and their closure closed half spaces.

Definition 6. The crooked half-space with vertex $\mathrm{p}$ and direction vector $\mathrm{v}$ is defined by:

$$
\begin{aligned}
& \mathrm{H}(\mathrm{v}, \mathrm{p})=\left\{\mathrm{p}+\mathrm{v} \in \mathrm{R}^{3} \mid\left\langle\mathrm{u}_{-}, \mathrm{v}\right\rangle>0 \text { if }\langle\mathrm{v}, \mathrm{v}\rangle>0,\right. \\
& \left\langle\mathrm{u}_{-}, \mathrm{v}\right\rangle\left\langle\mathrm{u}_{+}, \mathrm{v}\right\rangle>0 \text { if }\langle\mathrm{v}, \mathrm{v}\rangle=0 \text {, } \\
& \left.\left\langle\mathrm{u}_{+}, \mathrm{v}\right\rangle>0 \text { if }\langle\mathrm{v}, \mathrm{v}\rangle<0\right\} \text {, }
\end{aligned}
$$

and the corresponding crooked plane is its boundary $\partial \mathrm{H}(\mathrm{v}, \mathrm{p})$ [7].

Facts about closures: The closures of crooked planes in Minkowski patches are crooked surfaces.

We use Mathematica program to visualize crooked plane as following :

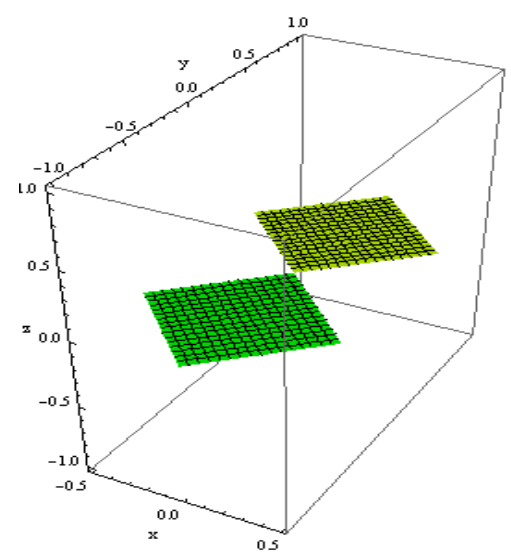

Figure 8. The stem in crooked plane

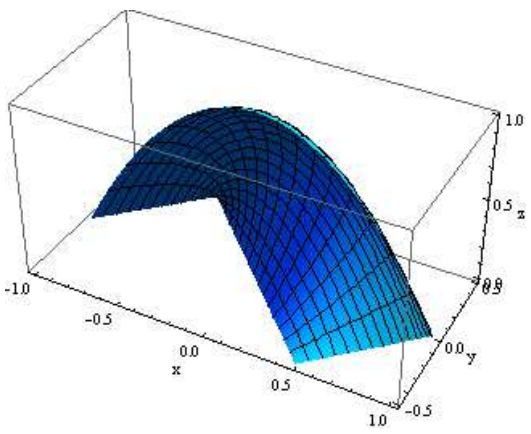

Figure 9. The first wing

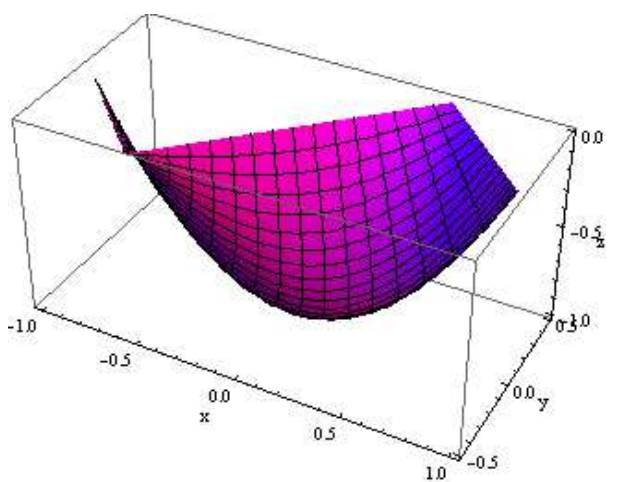

Figure 10. The second wing

Show $[\mathrm{S}, \mathrm{W} 1, \mathrm{~W} 2]$

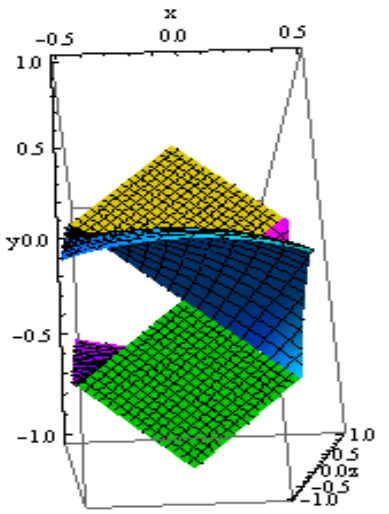

Figure 11. The crooked plane

\section{Conclusions}

This research deals with studying the properties of Minkowski patch which is the natural structure of Minkowski space. Also, we have been able to see the points and curves in Minkowski space. Furthermore, Visualized Minkowski patches embedded in $\mathrm{R}^{\mathrm{n}+1} \backslash\{0\}$.

\section{Acknowledgements}

I wish to express my gratitude towards to Professor Dr. William M. Goldman, University of Maryland and Distinguished Scholar-Teacher Professor, Department of Mathematics, for his valuable, guidance, patience and support. I consider myself very fortunate for being able to work with a very considerate and encouraging professor like him.

\section{Nomenclature}

$\begin{array}{ll}\operatorname{Ein}^{n, 1} & \text { Einstein Universe; Einstein Space } \\ \widehat{E \iota n}^{n, 1} & \text { The Double Covering } \\ \widetilde{E l n}^{n, 1} & \text { The Universal Covering } \\ R^{n+1,2} & \text { The Real inner product space of type }(\mathrm{n}+1,2) \\ E^{n, 1} & (\mathrm{n}+1) \text { Minkowski Space } \\ \tilde{p} & \text { Tilde }\{\mathrm{p}\} ; \text { Slash [p) } \\ \widehat{p}, \bar{p}, p^{\prime} & \text { Hat }\{\mathrm{p}\}, \text { Bar }\{\mathrm{p}\}, \text { Prime }\{\mathrm{p}\} \\ \oplus & \text { The Direct Sum; Xor; Bigoplus }\end{array}$



$\approx \quad$ Is isomorphic to
L(p) Light cone

MSC: 14Rxx, 51B20.

\section{References}

[1] Izumiya, S. and Saji, K., The mandala of Legendrian dualities for pseudo-spheres in Lorentz-Minkowski space and "flat" spacelike surface, Journal of Singularities,Vol.2, (2010), $92-127$.

[2] L'opez, R., Differential Geometry of Curves and Surfaces in Lorentz-Minkowski space, Mini-Course taught at the Instituto de Matem'atica e Estat'istica (IME-USP) University of Sao Paulo, Brasil, October 18, 2008.
[3] Izumiya, S. and Yıldırım, H., Slant Geometry of Spacelike Hypersurfaces in the Lightcone, Journal of the Mathematical Society of Japan, Vol. 63, Number 3 (2011), 715-752. lp

[4] Goldman, W. M., Crooked Surfaces and Anti-De Sitter Geometry, arXiv:1302.4911[math.DG] from Cornell University Library, February 20, 2013.

[5] Barbot, T., Charette, V., Drumm, T., Goldman, W. M., and Melnick, K., A primer on the $(2+1)$ Einstein universe, Recent Developments in Pseudo-Riemannian Geometry journal, European Mathematical Society, (2008), 179-230.

[6] Drumm, T. and Goldman, W. M., The geometry of crooked planes, Journal of Topology Vol. 38 (1999), No. 2, 323-351.

[7] Burelle, J. P., Charette, V., Drumm, T. and Goldman, W. M., Crooked Halfspaces, arXiv:1211.4177 [math.DG] from Cornell University Library, October 3, 2012. 